\title{
Design Implementation of E Purchase Browser Plug-in and the Issue on Browser Open Platform
}

\author{
Weichun Gao ${ }^{1}$, Weihai Gao², Wuxue Jiang ${ }^{3}$, Guidong Zhang ${ }^{1}$, Junfan Liu ${ }^{1}$ \\ ${ }^{1}$ School of Computer, Shenzhen Institute of Information Technology, Shenzhen, 518172, China \\ ${ }^{2}$ Meiyugao Culture Media co., LTD, Shijiazhuang, 05000, China \\ ${ }^{3}$ Department of Computer Engineering, Dongguan Polytechnic, Dongguan, 525000, China
} Keywords: E purchase, Browser plug-in, System design and implementation, Browser open
platform.

\begin{abstract}
E purchase is an application constructed on browser open platform, and it overcomes the limitation of existing technology in integration aspect of webpage and instant message, so, it can let common users establish chat room by self based on any webpage and have a chat with users browsing the same webpage. Furthermore, E purchase keeps away from social field of acquaintance chat, so that the net citizen can establish chat cluster by interest graph and opportunity webpage. It satisfies the instant communication of net citizens who browse identical product webpage.
\end{abstract}

\section{Introduction}

The enormous population base of China promotes the flourish of internet economy. The user scale of online shopping in China had reached to 277 million, and the average daily unique users of group purchase in China kept around 37 million by June, 2013. The enormous population promotes the flourish of e-commerce, but it also appearances new problems. Due to that there is no way to see commodity entity on network and to experience and inspect on the spot, so $88.0 \%$ of online shoppers are afraid of product quality and related service, then they hope to talk about the commodity on webpage with other users before shopping, and also hope the webpage can provide such an open communication platform. So as to avoid wasting the users' time and energy as the result of exchanging or returning goods caused by quality or service, and save the user cost; meanwhile, the users also hope to communicate with other users who are interested in the same product, so as to form group purchase and buy desirable products with lower price; besides, it can gathers the same demand of users by excavating mass of data, so as to form reverse $c 2 b$ individual group purchase and allow merchants to produce in accordance with the user need.

It has realized the integration of webpage and instant message gradually in aspect of idea and technology throughout global instant messaging software product, but, some of them are just confined to commerce website, while some of them can only be accomplished by webmaster at server end and can not allow common users to establish chat room by self at client end, so all of them can not fully satisfy the participation requirements of users. However, E purchase browser plug-in can provide plug-in for users through browser open platform, and it inserts instant messaging function in browser, so that the common users can establish chat room for any webpage[1]. By this way, it extends the function of browser, namely that it can not only allow users visiting the same commodity webpage to conduct group communication, but also can allow Ebusiness to participate in, to master user demand and form $\mathrm{c} 2 \mathrm{~b}$ production and sales.

Design of E Purchase Browser Plug-in

E purchase makes it possible for any common users to seek and add for any website and webpage, as well as enter chat room, meanwhile, it allows all of the users browsing the same website and URL to enter into the same chat room for communication, so as to provide communication site for all users [2]. When common users long for communicating the webpage content with other user group during webpage browsing, users can bind a chat room for this webpage through this product or seek the chat room bound in this webpage through this product, and users can enter into the chat room to communicate with other users if there is chat room; at the same time, users also hope to 
communicate with other users with interest to the same product, so as to form group purchase and buy the desirable product in lower price; moreover, it can collect the same kind of demand of users by chat room communication, so as to form reverse $\mathrm{c} 2 \mathrm{~b}$ individual group purchase and allow merchants to produce in accordance with the user demand.

Functional structure of E purchase browser plug-in. E purchase browser plug-in includes three parts of client-side, server-side and database [3].

Design development of client-side: client-side software can obtain the URL of current browser, send seek instruction of the described URL chat room to server-side, adopt the search results about URL chat room from server-side, and display the described search result to the window for users to enter into by selection; it also includes information exhibition bound by chatting URL, users' detailed information viewer, chat room information search bound by URL, chat room bound for URL, etc..

Design development of server: it makes it possible to interact information with client-side, including seek instruction about URL chat room sent by client-side, function of looking up the chat room of described URL from database and returning the search results to client-side [4]. It also includes function of adopting the establishment instruction of URL chat room and storing the described URL, account information, etc. of chat room in database.

Design development of database: database is mainly used for storing the chat room of URL, including URL table, chart room table of URL, users; table, etc.. Database adopts the seek instruction about URL chat room, and can seek related chat room from the table, furthermore, it can also add and delete chat room, etc..

Business process of system is shown in Fig. 1.

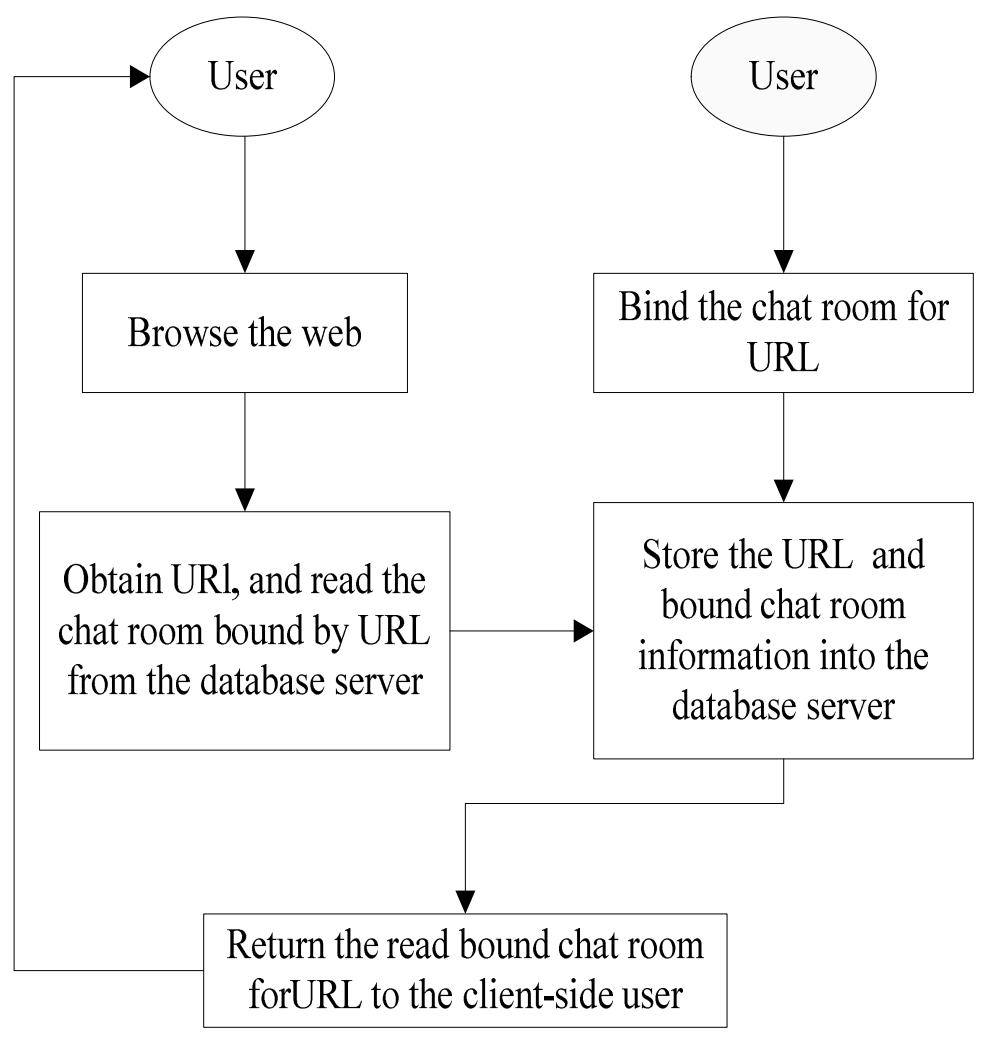

Fig. 1: System business flow chart

Login operation procedure of system client-side users. The following procedure needs to be conducted when users $\log$ in this system:

1) Identity authentication. Only when user name and password are correct can users log in instant messaging system of this webpage.

2) Users can bind, delete and seek chat room for URL, as well as manage personal information. 
The operation procedure for users to log in the instant messaging system of this webpage can be drawn by using UML after the analysis of above function.

\section{Realization of $\mathbf{E}$ chat browser plug-in}

The key technology for the realization of E purchase browser plug-in includes the acquisition of current browser URL, the encryption of database URL, and the method of resolving character string of URL.

1) Obtain the URL of current browser. The popular browsers at present are IE, Firefox, etc.. The kernels of the two kinds of browser are different, so their methods of obtaining URL are different also. It obtains URL through window handle method, and the process of obtaining current URL is shown in Fig. 2.

2) Md5 encryption of URL in database. Due to that the character string length of URL is big and the character is in disorder, it makes use of md5 encryption in order to make the character string of URL become short and easy for searching, as well as improve the efficiency. The detailed process is shown in Fig. 3.

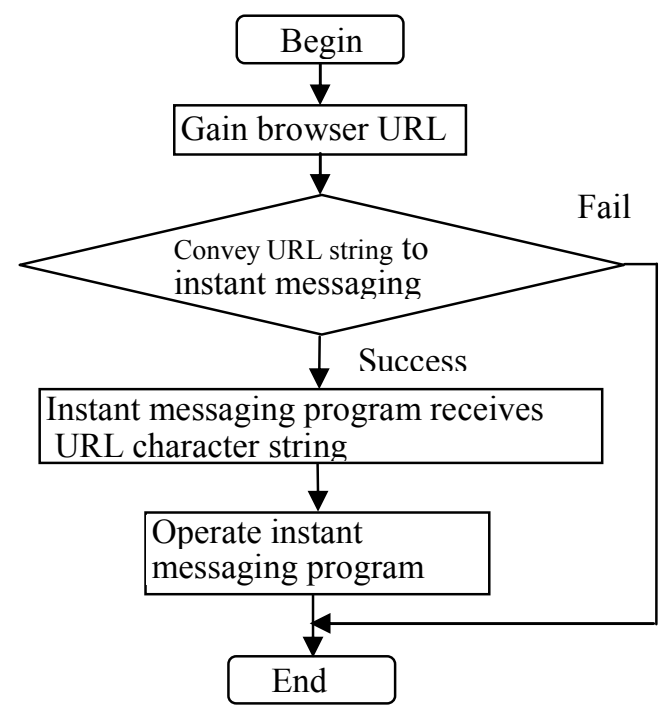

Fig. 2: the URL of current browser

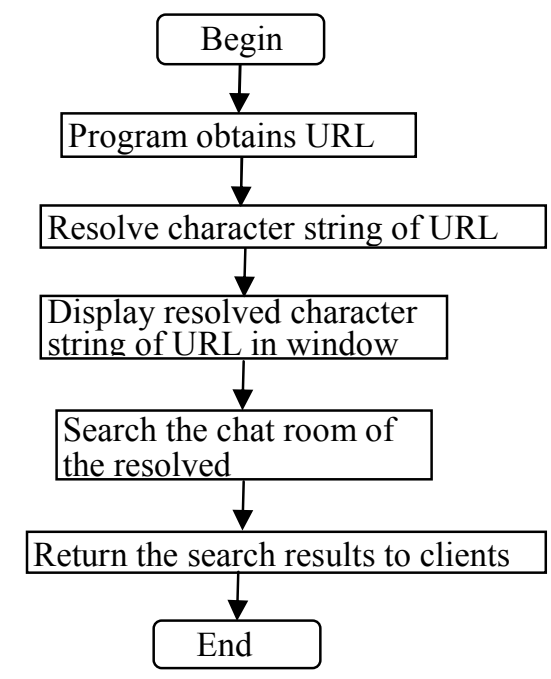

Fig. 4: Technique of resolving URL browser plug-in

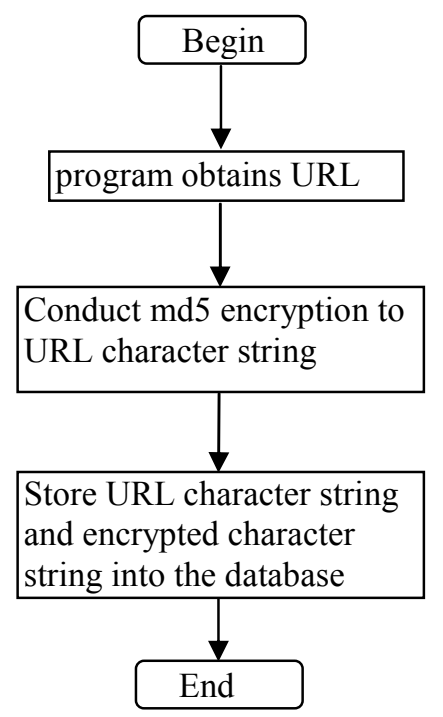

Fig. 3: encryption of URL in database

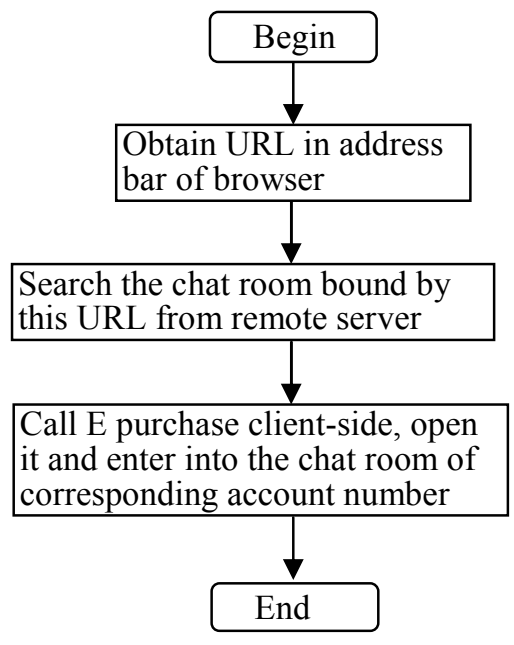

Fig. 5 Execution step of E purchase character string 
3)Technique of resolving URL character string. URL character string is marked by "/" character which resolves the current browser URL into multiple URLs. Such as that it can resolve URL http://www.mmllq.net/bbs as the two URLs of http://www.mmllq.net/ and http://www.mmllq.net/bbs, and then users can search chat room of these URLs separately from database. The detailed process is shown in Fig. 4.

\section{Issue of E Purchase Browser Plug-in on Browser Open Platform}

In internet era, the behavior of encapsulating URL service as a series of data interfaces to open to the outside for the usage of the developer of the third party is called Open API. The platform offering Open API is called open platform. The browser open platform allows the platform provider and the developer of the third party to achieve mutual benefit and interest apportionment [5]. Issue E purchase to browser open platform, then it can be dragged to toolbar of browser after being approved, so that when in need, users can click on E purchase plug-in icon and the browser plug-in can complete the function shown in Fig. 5.

\section{Conclusion}

E purchase is an application constructed on browser open platform, and its function is to let users establish chat room by self based on any webpage, so as to chat and communicate with users browsing the same webpage. E purchase keeps away from social field of qq acquaintance chat, and net citizens can establish chat group by interest graph and opportunity webpage contents. So it satisfies the instant communication of net citizens who browse identical product webpage.

\section{Acknowledgements}

This work was financially supported by the project of Guangdong college students' entrepreneurship training program (No.1295713017), and by Guangdong province higher vocational education teaching reform project (No. 20130201095), and by the project of China higher vocational education research association(No.GZYLX1213279), and by the project of Guangdong higher vocational education research association(GDGZ14Y07), and by the key teaching reform project of Dongguan Polytechnic (No. JGXM2014020), and by the fund project of Dongguan Polytechnic(2014d16, 2014a06).

\section{References}

[1] Qin Bian, Sandra Forsythe. Purchase intention for luxury brands: A cross cultural comparison. Journal of Business Research, 65(10), pp.1443-1451, 2012.

[2] Sameer Patil, Alfred Kobsa. Enhancing privacy management support in instant messaging. Interacting with Computers, 22(3), pp. 206-217, 2010.

[3] Shirley O'Sullivan. Instant Messaging vs. instant compromise. Network Security, 2006(7), pp. 46, 2006.

[4] Syed H. Akhter. Digital divide and purchase intention: Why demographic psychology matters. Journal of Economic Psychology, 24(3), pp. 321-327, 2003.

[5] Yang Zeng, Bing Wang, Yongtao Li and Rong Huang, 2014, Oxidation-Neutralization precipitation method in treatment of electroplating copper wastewater, Journal of applied science and engineering innovation, Vol.1 No.2, pp.174-178.

[6] Jeremy J. Sierra, Michael R. Hyman. Outlet mall shoppers' intentions to purchase apparel: A dual-process perspective. Journal of Retailing and Consumer Services, 18(4), pp.341-347, 2011. 